\title{
Precision Agriculture and Robotics
}

\author{
Reshma Rajmane \\ B.E. Mechanical Engineering, \\ Pune, India
}

\author{
Nikhil Gitay \\ B.E. Mechanical Engineering, \\ Pune, India
}

\author{
Aarya Yadav \\ B.E. Mechanical Engineering, \\ Pune, India
}

\author{
Yash Patil \\ B.E. Mechanical Engineering, \\ Pune, India
}

\author{
Apurv Sonawane \\ B.E. Mechanical Engineering, \\ Pune, India
}

\begin{abstract}
Identifying specific requisite according to field design and management, analyzing those and finding out the most appropriate and best solution, using information and technology is precision agriculture. Maximizing profits, reducing wastage of resources such as fertilizer and manpower are features of it. In this method of farming, advanced and updated technology tools and devices such as GPS and remote sensing are used to make the precise decision while performing agricultural tasks. Its main objective is not having the same production everywhere but to manage and evenly divide resources available. This can be a solution to the problems faced by farmers globally all around. This paper focuses on comparing and analyzing precision agriculture against traditional techniques and tools. Robots used for agriculture purposes are known as 'Agribot' or Agricultural robot. This paper focuses on various robotics tools used in agriculture for performing agricultural tasks.
\end{abstract}

\section{Keywords-Precision agriculture, agribot, robot $\mathrm{Oz}$}

\section{INTRODUCTION}

India's 55\% population depends on agriculture for its livelihood and this percentage may keep decrease in upcoming future because of less productivity, continuously decreasing farm field size, various crops diseases, unpredictable natural disasters etc. Non-availability of skilled labour, increase in labour wages makes conditions worse. Keeping in mind the ICAR vision 2020 - "To harness science to ensure comprehensive and sustained physical, economic and ecological access to food and livelihood security to all Indians, through assessment, refinement, generation and adoption of appropriate technologies." Currently, India is moving towards precise agriculture. [1] Various aspects of agriculture and identifying as well as managing available resources, according to field size in specific agricultural land, composition of soil, economic and environmental conditions, and coming up with a most appropriate solution using information and technology is Precision Agriculture. The main aim behind introducing this is increasing agricultural profit with minimum inputs, reducing wastage of fertilizers, herbicides \&man power. In this method of farming, advanced and updated technologies such as GPS, stereo vision cameras, remote sensing and most importantly robotics are introduced to make it more precise and productive.

\section{NEED FORPRECISION AGRICULTURE IN INDIA}

Although the Indian government designed and implemented various schemes for making agriculture more sustainable, more productive and climate-resilient and remunerative by introducing a golden fusion of information technology and space technology (satellite). This technology mainly focused on soil and conservation measures, efficient water resource management, efficiently predicting upcoming disasters. Still as compare to developed nations India's agriculture production is still low.
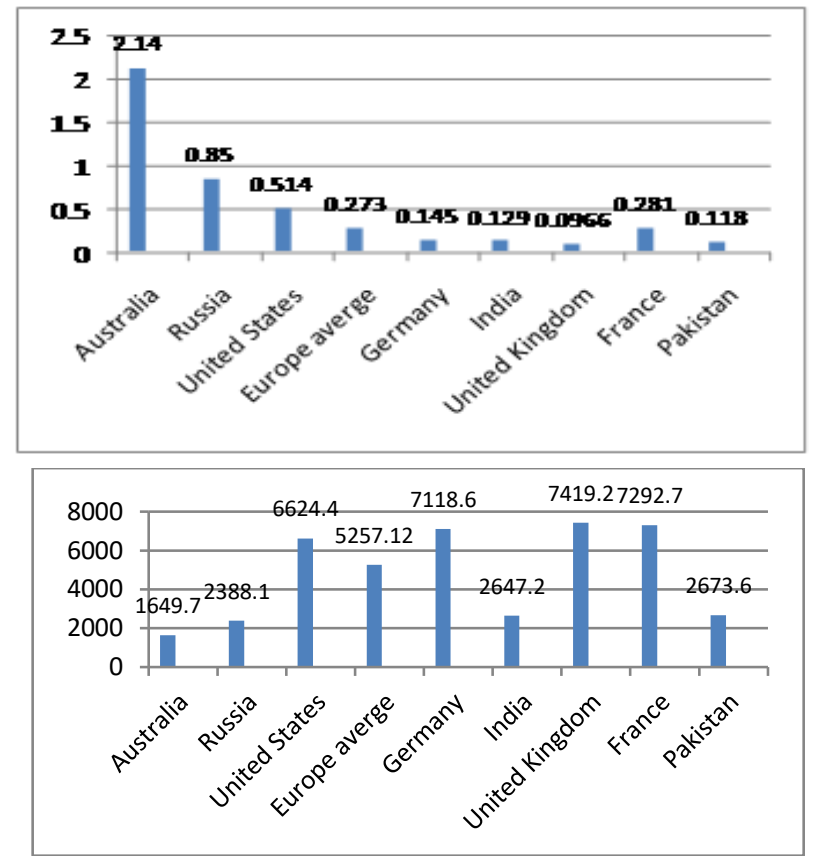

Fig. 1. Comparison between countries based on land distribution and production [2]

The Bar graphs below show a comparison between various countries based on land distribution in terms of a hectare per capita and production in $\mathrm{Kg}$ per hectare. From the data above it is clear that developed countries lead production capability because of precision agriculture whereas India lagging because of conventional approach it made in agriculture. Hence India needs to move towards precision agriculture. 
III. CONVENTIONAL AND PRECISION AGRICULTURE

Conventional agriculture vs Precision Agriculture

\begin{tabular}{|c|c|}
\hline $\begin{array}{l}\text { Conventional } \\
\text { Agriculture }\end{array}$ & $\begin{array}{c}\text { Precision } \\
\text { Agriculture }\end{array}$ \\
\hline \multicolumn{2}{|c|}{ Choose a good location } \\
\hline Manually & Using drones and GPS \\
\hline \multicolumn{2}{|c|}{ Soil Preparation } \\
\hline $\begin{array}{l}\text { Adding chemicals based on } \\
\text { previous experience }\end{array}$ & $\begin{array}{c}\text { Using sensors like temperature } \\
\text { sensor, Humidity Sensor, Volatile } \\
\text { matter sensor etc. }\end{array}$ \\
\hline \multicolumn{2}{|c|}{ Field preparation (Ploughing and planking) } \\
\hline Using tractors and bullocks & $\begin{array}{l}\text { Agricultural robot for automatic } \\
\text { ploughing }\end{array}$ \\
\hline \multicolumn{2}{|c|}{ Seeding and planting } \\
\hline Manually using hand tools & $\begin{array}{l}\text { Precision drills, Broadcast seeders, } \\
\text { Seed drills, Air seeders }\end{array}$ \\
\hline \multicolumn{2}{|c|}{ Watering } \\
\hline Drip Irrigation & $\begin{array}{c}\text { Drip Irrigation using Internet of } \\
\text { Things }\end{array}$ \\
\hline \multicolumn{2}{|c|}{ Fertilizer and Pesticide application } \\
\hline Hand spray and manually & $\begin{array}{c}\text { UAVs and UGVs, Sprayers, GPS, } \\
\text { Smartphone Applications, and } \\
\text { Remote sensing }\end{array}$ \\
\hline \multicolumn{2}{|c|}{ Weed removal } \\
\hline Using hand tools & $\begin{array}{l}\text { Blue River Technology and Naio } \\
\text { Technologies Weeding Robot Oz }\end{array}$ \\
\hline \multicolumn{2}{|c|}{ Harvesting } \\
\hline Manual picking & $\begin{array}{l}\text { Robotic pick and place arm, } \\
\text { Mechanical harvesting, limb shaker, } \\
\text { canopy shaker, Abscission Chemical }\end{array}$ \\
\hline
\end{tabular}

\section{STEPS PRECISION AGRICULTURE}

\section{A. Locating land position:}

Based on water, labour, transport \& market connectivity land position is chosen. For this GPS, data analysis techniques and past experience are taken into consideration. Whereas in India due to limited land availability enough importance is not given to this factor.

\section{B. Soil Sensing (Preparation):}

Usually, soil sensing is carried out to determine the constituents of soil what it requires in real-time. Initially, the nutrients are found by taking samples of soil from the fields in the lab and treating it with chemicals. The real part of soil sensing comes into picture during cultivation. Technology holds an immense advantage in today's agriculture. Soil sensing is carried out by drones or robots loaded with sensors moving across the fields.

1) Sensors: All these sensors are connected to a microcontroller to process the data obtained. The microcontroller processes the data, analyses, stores as well as sends to the operator. The microcontroller is controlled by a remote device over a network of frequency within the law. These devices are quite simple to use and can be handled easily by a poor educational background person also. Soil sensing works accurately when bulks of data are available for comparison and obtaining results.

Also, this technology can be used effectively in the irrigation process, by sensing dry parts of the field and providing the water to the same via drip irrigation.
2) E-Nose: Electronic Nose sensor is being used to determine the metallic fumes from the soil, and thus identifying the mineral deposits in the soil. The use of sensors may vary according to the crop being cultivated. These sensors are enclosed in a chamber and have electrical fans mounted which senses the odour with a change in airflow and locates the exact source of the odour.[3]



Fig. 2. Block diagram of the portable electronic nose system. [3]

\section{Ploughing and planking:}

Ploughing is the process of breaking, loosening the soil and turning it over for uprooting weeds and aerating the soil. Ploughing helps to improve nutrient values of the soil through loosening and turning of soil. It improves air circulation in order that roots can respire easily. Ploughing improves the water arresting capability of the soil. Planking is completed to crush the hard clods to smoothen the soil surface and to compact the soil lightly. Thus, the sector is formed ready for sowing after ploughing by harrowing and planking.

1. Agricultural Robot for automatic ploughing: A robot capable of performing operations like automated ploughing, seed disbursing, fruit picking and pesticide spraying. It also provides manual control when required and keeps tabs on the humidity with the assistance of humidity sensors. The most essential component here is that the AVR at mega microcontroller that supervises the entire process. Initially the robot tills the whole field and proceeds to plough, simultaneously dispensing seeds side by side. The device used for navigation is an ultrasonic sensor which continuously sends data to the microcontroller. On the farm, the robot operates on automated mode, but outside the sector is strictly operated in manual mode. The alerting mechanism is a GSM module that sends a text message to the farmer informing him about the breach in point. The farmer then responds via SMS to either activate the water sprinklers or ignore the alert.[4]

\section{Seeding and planting:}

In some cases, seeds are sown whereas in other plants prepared in nursery or greenhouse chambers are planted. 
1) Air-assisted Strip Seeder: The Hokuriku Research Centre has designed and manufactured an air-assisted strip seeder. The seeding base width of the seeder is 10 $\mathrm{m}$ (base width of one side is $5 \mathrm{~m}$ ). The seeds are transported to injection ports situated at the bottom via blower of the seeder. It deposits the seeds at a distance of $30 \mathrm{~cm}$. Because of its flexibility and speed, field efficiency rises to $70 \%$. Its technology may promote labour-saving and low-cost technologies for direct seeding culture in the near future.[5]

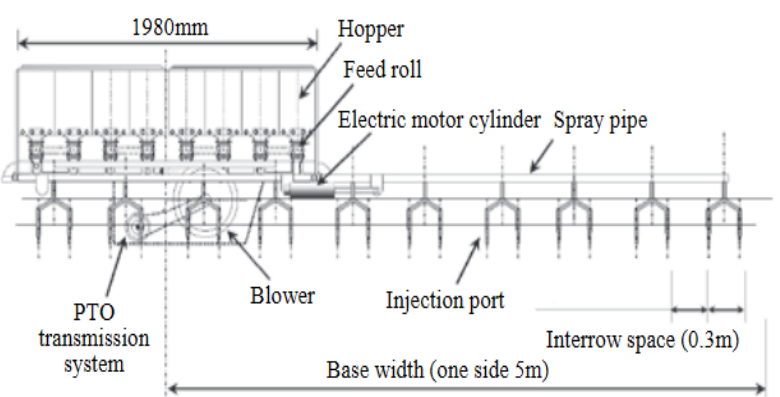

Fig. 3. Schematic diagram of air-assisted strip seeder [5]

2) Broadcast seeder: As there are various methods of sowing like- drilling, Seed dropping, Dibbling, Broadcasting is also one of these. Broadcast seeder is also known as broadcast spreader or broadcaster, or even centrifugal fertilizer spreader (Europe). It is a farm implement commonly used for spreading seed, lime, fertilizer, sand, ice melt, etc. and is an alternative to drop spreaders/seeders. It is used particularly in establishing dense plant spacing. In comparison to traditional drilling planting or row sowing broadcast seeding is much faster, simpler, and easier. The major problem faced in this method is that there is no even distribution of seeds which eventually result in their decreased efficiency and thus is a wasteful process.

3) Seed drill: Seed drill is a tool which is used to sow seeds for crops at proper positions in soil and bury them to a specific depth. This guarantees that seeds are completely covered by soil. Before this method, farmers planted the seeds for cereal crops by carrying the seeds in a bag and walking up and down the field throwing or broadcasting the seed. The difficulties faced in this method are overcome by using seed drills. This machine aids in proper distribution of seeds between rows which can be set by the manufacturer. However, with enough sunlight, nourishment and water supply it resulted in increased productivity.

E. Watering:

Water is the most important resource for agriculture. By constructing artificial water reservoir water is supplied to farms. In precision its quantity is control.

1. Drip Irrigation using Internet of Things: In the proposed system first on the AC supply it goes to the power unit then first check action is performed $\mathrm{ON}$ or OFF. Supposed action is on then it set the water pump. When action is off the power unit goes to the raspberry pi to perform the action. Then raspberry pi sends to a sensor for sensing the value by using the internet module. Then sensor sense that value and send these values to the raspberry pi. Then raspberry pi sends these values to the web-server. And then the web-server compares the throughput value of the sensor and current value which send to the raspberry pi. And send these comparable values to raspberry pi then raspberry pi perform the action.[6]

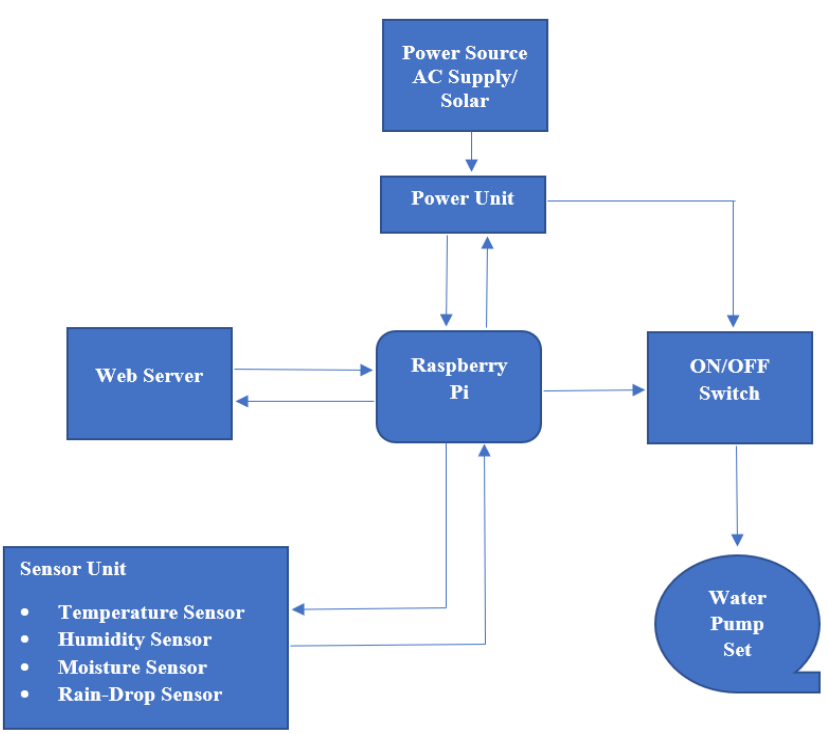

Fig. 4. Block diagram of drip irrigation [6]

\section{F. Fertilizer and Pesticide application:}

As humans are affected by germs and infections, crops also get affected by pests and are liable to diseases. So, there is a need to prevent, cure and detect pests and diseases. Using precision agriculture techniques pests and diseases can be controlled and eliminated effectively. Tools used in precision agriculture are UAVs, UGVs, Patch sprayer, Canopy sprayer, GNSS and Mechanical/Thermal tools.

1. Unmanned Aerial Vehicles and Unmanned Ground Vehicles: UAVs and UAVs are controlled remotely by pilots. These vehicles are attached to different equipment for a variety of purposes. UAV flown over the farm field captures high-resolution images of any pest, diseases, rogue plant or potential threats to the crop, which allows farmers to gain oversight of nutritional or protection requirements of crops, using infra-red cameras and other visual capturing mediums, potentially saving them time and money by allowing for precision applications of chemicals, fertilizers or crop irrigation. Multispectral and hyperspectral imagery are often used to measure the responses of plants in the visible and near infra-red parts of the electromagnetic spectrum to remotely detect plant stress. With the help of UGVs biodegradable and non-biodegradable wastes are removed from the fields. Furthermore, sprayers are attached to UAVs and UGVs to spray pesticides and chemicals on the fields, which cures and prevents the increase of diseases. Sometimes, these vehicles are also used to release beneficial insects over crops, this method is termed as biocontrol. 
2. Sprayers: Sprayers are used to spray fertilizers on the crops. But in precision agriculture, instead of squirting fertilizers all over the field, it is sprayed at a specific region of the field or a specific part of the crops, at a controlled rate, based on the data gathered from others tools. For instance, a spraying system built around a quadruple nozzle body, which is integrated with GPS navigation systems through the software and will independently control an unlimited number of nozzles along with a boom. In this way pesticides are sprayed on affected regions only and at a desirable rate. Moreover, precision sprayers eliminate overapplication on the slow end of a turning boom, and under-application on the fast end. Along with variablerate capabilities, the spraying system is able to control spray particle size and keep spray pressure stable over a range of speeds by switching nozzles as the sprayer speeds up and slows down.

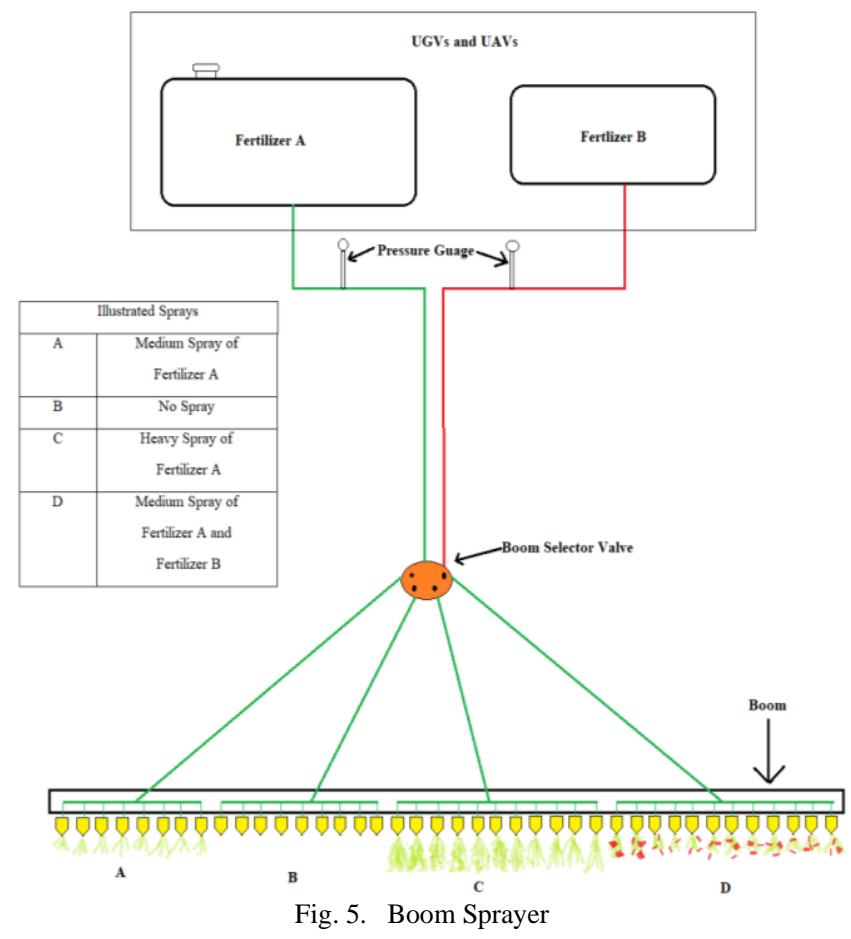

3. Smartphones application: Researchers from the University of Lincoln, UK, are developing a new smartphone app to help farmers tackle the pests destroying their crops, and it could soon have a major impact on the way information about the natural world is gathered, stored and accessed worldwide. A team of researchers is designing and building the specialist app to help farmers in hot climates identify and record the spread of locusts on their land. The software identifies various pests via the smartphone's camera and will be able to identify the phase of the insect's growth and register its location through the phone's IP address. This information can then be accessed by the farmer so that they can use pesticides more precisely and disrupt the growth of pests in the initial stages of their lifespan, which results in a significant reduction in the amount of crop damage.[7]
4. GPS: The Global positioning system (GPS); is a network of about 30 satellites orbiting the Earth at an altitude of $20,000 \mathrm{~km}$, which transmits and receives real-time data. This real-time data collection gives accurate position information, which in turn leads to efficient analysis and manipulation of large amounts of geospatial data. Precision agriculture is all about gathering timely geospatial information on soil and plant requirement and prescribing site-specific actions to protect the crops and increase agricultural yields. GPS is a crucial tool of precision agriculture which is used in different agricultural processes.

\section{G. Weed removal:}

Weed control is a significant issue in agricultural crop production. Weeds battle with crop plants for moisture, nutrients and sunlight and can have an adverse impact on crop yields and quality if not controlled. Weed mapping is the process of recording the position, density (biomass) and preferably species of various weeds using machine vision. Robotic advanced technology can also provide a way of reducing agriculture's present dependence on herbicides, improving its sustainability and reducing its environmental impact.

1) Blue River Technology: Blue River focuses on applying machine learning, robotics, and computer vision technology to agricultural equipment that will, in turn help farmers in making better decisions. Their primary product, See \& Spray, uses computer vision, machine learning, and advanced robotic technology to distinguish between crops and weeds and then spraying only the weeds. Farmers manage large networks of fields and millions of plants - in order to better maximize their yields they need to revaluate their herbicide distribution on a plant-by-plant basis. Farmers will typically employ the "one size fits all" approach, spraying their entire fields with a single uniform solution.

2) Naio Technologies Weeding Robot Oz: Oz follows the crop rows of the farm and guides itself. Oz can weed all of your crop rows without human supervision. The $\mathrm{Oz}$ robotic weeder comes with a configuration unit that permits you to select the proper operating mode for you. The unit will also ask you to enter specific information on the plot $\mathrm{Oz}$ is required to weed (number of rows, plot size, etc.). When in autonomous mode, the robotic weeder will send a text message to inform its owner when it finishes weeding. It is also capable of detecting potential anomalies during work (obstacles, blocked tools, errors in row length), in which case it will use text messages to send alerts.

\section{H. Fruit Harvesting:}

One of the most labour-intensive tasks of agriculture is fruit harvesting as a method of harvesting varies with crops. Height of crop, the shape of fruit etc factors are analysed while using harvester. 
1) A robot with 4 Degree of Freedom Manipulator:Production of Strawberry occupies a greater portion of fruit economy in various countries such as Japan. For these types of fruits, this robot was invented. It mainly consists of a square led-array and stereo vision cameras. Two cameras are mainly used for identifying and localizing the position of fruit on the stem. After the storing position data of fruit, the end effector is situated next to fruit. While the third camera is provided for detecting the stem of fruit and its orientation. This data is analysed and processed by the processor, and according to its orientation of the end, the effector is changed with the help of the tilting mechanism and process of fruit harvesting proceeds. Once the stem is grabbed by the end effector the stem is cut by cutters mounted on the grasping mechanism. The flexible and compatible mechanism takes the strawberries to a tray. This procedure is carried out for all the detected strawberries.

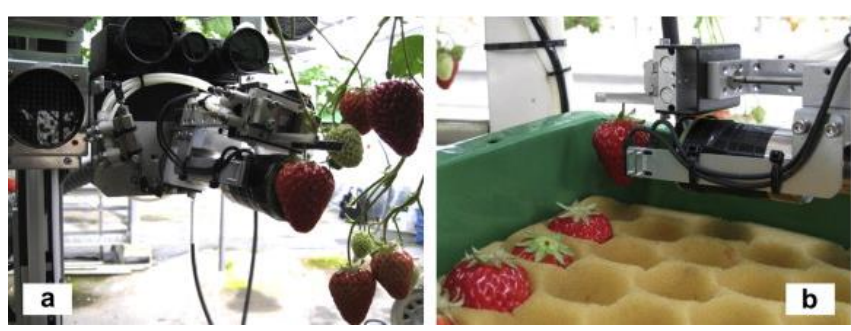

Fig. 6. Fruit Harvesting

2) Mechanical harvesting: In mechanical harvesting method is mainly used for citrus fruit. In this tree is mechanically shaken so that fruits drop from the tree in allocated containers and there will be minimal damage to the tree.

- Canopy Shaker: In this vertical shaking of secondary limbs is conducted by a shaker. This method is particularly used for removing excessive immature oranges during summer.

\section{TOOLS USED IN PRECISION AGRICULTURE}

\section{A. Global Positioning System:}

The Global positioning system (GPS) is a network of about 30 satellites orbiting the Earth at an altitude of 20,000 km, which transmits and receives real-time data. This real-time data collection gives accurate position information, which in turn leads to efficient analysis and manipulation of large amounts of geospatial data. Precision agriculture is all about gathering timely geospatial information on soil and plant requirement and prescribing site-specific actions to protect the crops and increase agricultural yields. GPS is a crucial tool of precision agriculture which is used in different agricultural processes.

\section{B. Drones:}

Drones (UAVs) are used in agricultural activity to prevent, cure and detect pests and diseases of the crops. Like any other aerial vehicles, UAVs also have pilots, which control the drones from the ground. Drones are attached with different equipment for usage purposes UAV flown over the farm field captures high-resolution images of any pest, diseases, rogue plant or potential threats to the crop. This aerial imagery helps to manage agricultural activity more effectively. This technology allows farmers to gain oversight of nutritional or protection requirements of crops, using infra-red cameras and other visual capturing mediums, potentially saving them time and money by allowing for precision applications of chemicals, fertilizers or crop irrigation. Multispectral and hyperspectral imagery are often used to measure the responses of plants in the visible and near infra-red parts of the electromagnetic spectrum to remotely detect plant stress. Furthermore, sprayers are attached to UAVs, to spray pesticides on the fields, which cures and prevents the increase of diseases. Sometimes, drones are also used to release beneficial insects over crops, this method is termed as biocontrol.

\section{Stereo Vision Camera:}

The human eye has an inbuilt binocular vision due to which we can see objects in-depth view easily and differentiate between various objects easily. The human binocular vision perceives depth by using Stereo disparity, which refers to the difference in image location of an object seen by the left and right eyes, resulting from the eyes' horizontal separation. The brain uses this binocular disparity to extract depth information from the two-dimensional retinal images, known as stereopsis. Hence, Stereo vision camera is nothing but a depth-sensing tool used to identify different objects easily, which is not possible with a 2D camera. This camera has a setup of two lenses like a human eye. The camera works on the principle of triangulation. There are two main types of Stereo Vision Cameras present in market Passive and Active. The first one does not need a projection of light to work while the latter needs a projection of light to work.

\section{ADVANTAGES OF PRECISION AGRICULTURE:}

- A solution to lack of agricultural labour: In developing as well as in developed nations one of the most prominent problems faced by the agriculture sector is low or non-availability of an efficient and productive workforce. With the robotics, more precise and effective labours will be introduced in agriculture as robots can work without fatigue and more accuracy. This factor can be witness Robots as working in the agriculture sector is not a new concept for developed countries which includes the U.S.A., Japan, China etc. According to the International Federation of Robotics, Japan Robot Association no. of robots used in various sectors worldwide is shown below: This data showcases the variation and modes, the changes it will bring out in agriculture in the upcoming decade as expenses on agricultural robots are increasing rapidly. Robots are not only a reliable workforce but also cheap labour excluding initial investment it required.

- Variety of delicate crops can be preserved and grown: Many agricultural scientists and companies are collaborating together to develop robots which can take care of plants as human do. For example, 
Fendt's Xaver Robot, which is expected to launch in the upcoming year, it is precisely design and develops for the corn crop. It not only plants the crop but also performs other agricultural activities too. Varies types of robots designed for fruit harvestings such as apple picker, strawberry and citric fruit harvester are some examples where robots occupy the position of human and perform the task with more perfection.

- Robotics will increase crop yields: Using technological advancements as well as increased use of robotics in precision agriculture will definitely aid in boosting the yields of crops. For insistence, human interference can be lowered by using robotic vehicles picker. Even, various plant diseases, insects as well as weeds would be noticed with the help of sensors. Besides, robots can easily do things like pesticide spraying in required amounts at required locations. The information from sensors can be collected by humans and analyzed for different further studies.

- Farmers will spend more time on the business of farming: Due to the ever increase in demand for food and shortage of labour, farmers are increasingly relying on robots in precision agriculture for their necessity. Such advancement in systems enables labour and time saving, along with increased crop yield. Thus, proving that technological progress is permeating all parts of our lives.

\section{LIMITATION OF PRECISION AGRICULTURE:}

- Robots require a lot of money to make/buy and maintain, to keep them running efficiently.

- Farmers can lose their jobs as agribots can change the culture or the sentimental appeal of agriculture.

- The agribots may be more acceptable to the nonfarm community. The jobs in agriculture require intelligence and quick and the robots can be substituted with the human operator.

VIII. FUTURE OF PRECISION AGRICULTURE:

1. Cropping Sensors: Almost centuries Indian farmers follow a group of prescribed manuals or guideless during fertilizer application and spraying. The local Agri university or research agencies give farmers data on the dosage and mode of application which is common to all or any farms and farmers. The evolution of crop sensors goes to vary in this scenario. Sensors tomorrow are getting to provide site-specific information on the precise amount of nutrient required for a farm. This will save tons of fertilizers and also conserve valuable soil resources.

2. Farming Vertically: Skyscrapers in agriculture will be the longer-term goals in increasing farm output per area. With draining soil fertility levels and increasing urbanization, land for farming tomorrow is going to be costlier than before. This means that food goes to be costlier and not affordable to folk. To avoid this example, the vertically farming technique using soil- less medium and using artificial lights are the longerterm solutions. This technique will reduce cost, produce more, protect crops from inclemency and ultimately produce food which is safe and healthier.

3. Robotic Swarms: Think of numerous agribots loaded with sensors collecting real-time information in the fields. The data recorded and shared to the cloud, analyzed and automatic reports are generated in terms of excess moisture, nutrient deficiency and many more insights of the farm. This idea is already in use in Israel farm to increase productivity. An international firm called FENDT is currently working on a project called MARS Mobile Agricultural Robot Swarms. It is research in the field of agricultural robotics funded by the European Union.

4. Big data in farming: Storing and analysing tons of data will help in making real-time decisions quickly and with greater accuracy and precision. Feedback on various plants such as their height colour and diseases and their life span will help to increase productivity in a particular farm or field or area. Using the above-mentioned technologies in Indian agriculture within affordable price will boost the Indian agricultural revenue. The above technologies are expected to be utilized on a large scale in India by farmers by 2022 .

\section{REFERENCES}

[1] Mondal, P., \&Basu, M. (2009). Adoption of precision agriculture technologies in India and in some developing countries: Scope, present status and strategies. Progress in Natural Science.

[2] Worldbank.org.in

[3] T. Pobkrut and T. Kerdcharoen, "Soil sensing survey robots based on electronic nose," 2014 14th International Conference on Control, Automation and Systems (ICCAS 2014), Seoul, 2014, pp. 1604-1609.

[4] J.Raja1, \&W.StanleyKarunakaran Automatic Ploughing And Seeding Robot, International Conference on Electrical, Information and Communication Technologies (ICEICT -2017)

[5] Furuhata, M., Chosa, T., Shioya, Y., Tsukamoto, T., Seki, M., \& Hosokawa, H. (2015). Developing direct seeding cultivation using an air-assisted strip seeder. Japan Agricultural Research Quarterly. Japan International Research Center for Agricultural Sciences.

[6] Gaikwad Tararani1, GanduleShital, KorabuSofiya, PawarGouri, Prof.Vasekar S.R. (2018) Smart Drip Irrigation System using IOT International Research Journal of Engineering and Technology (IRJET)

[7] Hayashi, S., Shigematsu, K., Yamamoto, S., Kobayashi, K., Kohno, Y., Kamata, J., \& Kurita, M. (2010). Evaluation of a strawberry-harvesting robot in a field test. Biosystems Engineering, 105(2), 160-171.

[8] Precision pest control: smartphone app is the farmer's newest weapon in crop protection (http://www.lincoln.ac.uk//news/2019/01/1507.asp _ -via @ lincolnlates 